\title{
Targeting polo-like kinase 1 in acute myeloid leukemia
}

\author{
Joseph M. Brandwein
}

\begin{abstract}
Polo-like kinase 1 (Plk1) plays a number of important roles in the passage of cells through mitosis. It is expressed at high levels in a variety of malignancies, including acute myeloid leukemia (AML). Inhibition of Plk1 results in cell cycle arrest and apoptosis, and has anti-tumor effects in pre-clinical models. A number of Plk1 inhibitors have been developed, some of which have entered clinical trials. Of these, volasertib (BI6727) has been most extensively studied clinically in AML. Volasertib has demonstrated antileukemic activity in $A M L$, both as a single agent and when combined with low-dose cytarabine. It is well tolerated, with the major toxicity being reversible myelosuppression. A recently completed phase III clinical trial in older AML patients will address the question of whether adding this agent to low-dose cytarabine is associated with a survival advantage.
\end{abstract}

Keywords: acute myeloid leukemia, chemotherapy, leukemia, polo-like kinases, volasertib

\section{Acute myeloid leukemia}

Acute myeloid leukemia (AML) is an aggressive hematologic malignancy characterized by a clonal maturation arrest at an early myeloid progenitor stage, and the proliferation of primitive myeloblasts. This results in the accumulation of blast cells in the bone marrow leading to severe cytopenias and associated complications. The median age of AML is approximately 70 and the frequency increases dramatically above age 60 (SEER, 2014).

The treatment of younger and more fit older patients consists of intensive induction chemotherapy, using cytarabine plus an anthracycline, to achieve a complete remission (CR), followed by several cycles of consolidation chemotherapy. $\mathrm{CR}$ rates are in the $70 \%$ range in patients under age 60 , and $50-55 \%$ in those over age 60 [Tallman et al. 2005; Grimwade et al. 2010; Mrozek et al. 2012]. However, the majority of patients will subsequently relapse. Cytogenetics and molecular mutations are important predictors of both $\mathrm{CR}$ and relapse with chemotherapy. Adverse risk cytogenetic groups include monosomal and complex karyotypes, as well as 3p26,17p and 11q23 abnormalities. CR rates with induction chemotherapy in these patients are in the $30-50 \%$ range, with 5-year overall survival (OS) of $10 \%$ or less [Grimwade et al. 2001, 2010; Gupta et al. 2005; Farag et al. 2006]. Among molecular mutations, fms-like tyrosine kinase internal tandem duplications (FLT3-ITD) are associated with a higher relapse rate and inferior survival [Mrozek et al. 2012; Linch et al. 2014], while nucleophosmin (NPM1) mutations are associated with a superior CR rate and OS [Schnittger et al. 2005; Mrozek et al. 2012]. Allogeneic hematopoietic stem-cell transplantation (HSCT) in CR can reduce the probability of relapse in poor risk patients, but many patients are not candidates for transplant owing to advanced age and comorbidities. Furthermore, the inability to achieve CR may preclude transplant, and success rates with HSCT are lower in poor risk patients [van Gelder et al. 2013]. In older patients, the 5-year OS with intensive chemotherapy is in the $10 \%$ range; for older patients with adverse risk karyotypes, there is little survival benefit with such aggressive treatment approaches, due to the low CR rates and brief CR duration [Gupta et al. 2005; Farag et al. 2006; Kantariian et al. 2006; Grimwade et al. 2010]. For patients with relapsed/refractory AML, results are dismal and have only marginally improved in recent years [Pemmaraju et al. 2015].
Ther Adv Hematol

2015, Vol. 6(2) 80-87

DOI: $10.1177 /$

2040620715571077

(c) The Author(s), 2015

Reprints and permissions: http://www.sagepub.co.uk/ journalsPermissions.nav
Correspondence to: Joseph M. Brandwein, MD, FRCPC

Division of Hematology, Department of Medicine, University of Alberta, 4-112 Clinical Sciences Building, 11350-83 Avenue, Edmonton, $A B$, Canada T6G 2 G3

jbrandwedualberta.ca 
In older patients who are not considered suitable for intensive therapy based on comorbidities and performance status, therapeutic options are limited. Management with supportive care alone, with hydroxyurea to control peripheral blast counts, is associated with a median OS of 3-4 months and 1-year OS less than $20 \%$ [Harousseau et al. 2009]. Low-dose cytarabine is widely used in such older patients. The MRC-15 trial demonstrated a CR rate of approximately $20 \%$ in such older patients, with an associated survival benefit compared with supportive care plus hydroxyurea [Burnett et al. 2007]. However, this benefit was restricted to patients with standard or favorable risk cytogenetics; those with adverse risk cytogenetics had no CRs and no survival benefit. Furthermore, even in more favorable risk groups CRs were always transient. Hypomethylating agents, including azacitidine and decitabine, have demonstrated activity in older patients with AML, and produce superior OS compared with best supportive care [Fenaux et al. 2010; Kantarjian et al. 2012; Dombret et al. 2014]. Response rates and OS with hypomethylating agents are at least comparable to those of low-dose cytarabine [Dombret et al. 2014]. However, these agents are not curative, and virtually all patients will eventually experience disease progression. Although many novel agents have been studied or are currently in clinical trials in older patients with AML, no agent has been demonstrated to produce a survival benefit to date compared with standard treatments. There is therefore an urgent need to develop and test novel therapies in such patients.

\section{Polo-like kinases}

Polo-like kinases (Plks) constitute a family of serine/threonine kinases which regulate several key steps in the passage of cells through mitosis. In mammals, four members of the Plk family have been identified, each of which contains both a serine/threonine kinase domain and a polo-box domain [Winkles and Alberta, 2005; Strebhardt, 2010]. Plk1 is the most extensively characterized of these. It is expressed primarily during the G2 and $\mathrm{M}$ phases of the cell cycle where it plays multiple crucial roles, including mitotic spindle assembly, transition from metaphase to anaphase, chromatid separation and the onset of cytokinesis [Dai et al. 2002; Hansen et al. 2004; van Vugt et al. 2004; Strebhardt, 2010]. Plk1 also plays a key role in regulating checkpoint controls, including activation of the cyclin-dependent kinase Cyclin $\mathrm{B} / \mathrm{Cdk} 1$ which controls entry into mitosis
[Chopra et al. 2010]. In addition, Plk1 phosphorylates the antiapoptotic protein Bcl-xl [Tamura et al. 2009]; inhibition of Plk1 decreases p-Bcl-xl expression, which may thereby promote apoptosis [Ikezoe et al. 2009].

Plk1 is expressed at very low levels in most normal tissues, including kidney, liver, brain, lung and pancreas [Winkles et al. 2005]. In contrast Plk1 is expressed at higher levels in tissues with rapidly proliferating cell populations, including placenta, spleen, ovary and testis [Winkles and Alberta, 2005]. Plk1 is overexpressed in a wide variety of cancers, including non-small cell lung [Wolf et al. 1997], breast [Wolf et al. 2000], ovarian [Weichert et al. 2004] and head and neck squamous carcinomas [Knecht et al. 2000], as well as melanoma [Strebhardt et al. 2000] and diffuse large B cell lymphoma [Liu et al. 2007]. Overexpression of Plk1 has been correlated with an inferior prognosis in several of these malignancies [Wolf et al. 1997; Knecht et al. 2000; Weichert et al. 2004].

Inhibition of Plk1 in tumor cell lines, using small interfering RNA or small molecule inhibitors, results in inhibition of cell proliferation with cell cycle arrest at the G2/M phase. This is associated with disorganized spindle appearance and an increase in histone $\mathrm{H} 3$ levels followed by apoptotic cell death [Liu and Erikson, 2003; Lenart et al. 2007]. Such inhibition has also been shown to reduce tumor growth in mouse xenograft models [Steegmaier et al. 2007; Renner et al. 2009]. Plk1 inhibition does not appear to be dependent on functional p53; in fact, there is evidence that Plk-1 mediated cytotoxicity is increased in cells with nonfunctional p53 [Craig et al. 2014].

Plk1 mRNA and protein have also been found to be overexpressed in multiple AML cell lines, as well as in blast cells from the majority of patients with AML [Ikezoe et al. 2009; Renner et al. 2009]. In contrast, Plk 1 expression in bone marrow and peripheral blood mononuclear cells, and in normal CD34-positive progenitor cells, is low [Ikezoe et al. 2009]. Expression in AML cells did not appear to correlate with cytogenetic subgroups in one study [Renner et al. 2009]. Inhibition of Plk1 by small interfering RNA or a selective Plk1 small molecule inhibitor in vitro is associated with a marked inhibition in proliferation of AML cell lines, accompanied by an accumulation of cells in the G2/M phase and induction of apoptosis. Incubation of fresh AML cells with a Plk1 inhibitor is also associated with a marked reduction in clonogenic growth. 
In contrast, normal CD34-positive progenitor cells, and bone marrow mononuclear cells, were largely insensitive to inhibition [Ikezoe et al. 2009; Renner et al. 2009].

In severe combined immunodeficiency mouse xenograft models, Plk1 inhibition resulted in a marked reduction in tumor volumes using the AML cell line HL-60 [Valsasina et al. 2012]. In the same study, using a murine model of disseminated leukemia using patient-derived AML cells, PLK1 inhibition was also associated with a prolongation in survival, and showed evidence of synergy when combined with cytarabine, with further prolongation in survival.

\section{Development of PLK1 inhibitors}

A number of different small molecule inhibitors of Plk1 are in preclinical and clinical development (see Table 1). GSK4611364A (GlaxoSmithKline, London, UK) is a highly selective thiophene amide inhibitor of Plk1 which induces mitotic arrest and inhibition of tumor growth in xenograft models. A phase I study has been completed in patients with solid tumors [Olmos et al. 2011].

Several newer Plk1 inhibitors have reported activity in preclinical studies. TAK-960 is a potent orally bioavailable selective Plk1 inhibitor which was shown to have activity against a variety of tumor cell lines, including K562 leukemia cells in vitro. This agent also produced marked growth inhibition of AML MV4-11 cells in a mouse xenograft model [Hikichi et al. 2012]. A phase I study has been completed in patients with advanced solid tumors, but has not yet been published. NMS-P937, another orally bioavailable agent, has been shown to have a high degree of activity and selectivity for Plk1 with a half maximal inhibitory concentration (IC50) of $0.002 \mathrm{umol} / \mathrm{L}$ [Valsasina et al. 2012]. It has produced marked inhibition of growth in both leukemia and colon carcinoma cell lines, and inhibition of acute monoblastic leukemia in a mouse model [Casolaro et al. 2013]. This agent has not yet been studied in humans.

BI2536 (Boehringer-Ingelheim, Ingelheim, Germany) is a highly potent Plk1 inhibitor that induces cell cycle arrest and apoptosis [Lenart et al. 2007; Steegmaier et al. 2007]. A phase I study with this agent has been reported in patients with solid tumors [Hofheinz et al. 2010]. When administered on a day 1 and 8 dosing schedule in this study, the maximum tolerated dose (MTD) was $100 \mathrm{mg}$; the doselimiting toxicity was grade 3-4 myelosuppression. In addition, a phase I/II trial of this agent was reported in older patients with relapsed/ refractory AML [Müller-Tidow et al. 2013]. The MTD was $200 \mathrm{mg}$ when given in a similar day 1 and 8 dosing schedule, and $350 \mathrm{mg}$ in a day 1 schedule, with the major grade 3-4 toxicities being myelosuppression with associated neutropenic sepsis. Of 54 evaluable patients, two achieved CR and three a partial response, for an overall response rate of $9 \%$. Evidence of cell cycle arrest was seen in AML blasts in vivo. Further development of this agent was subsequently discontinued in favor of BI6727 due to the latter's improved pharmacokinetic profile (see below).

\section{Rigosertib}

ON01910 (Onconova Therapeutics, Newtown, $\mathrm{PA})$, also known as rigosertib, is a non-adenosine triphosphate (ATP) competitive benzyl styryl sulfone analog that inhibits Plk1 as well as PI3Kinase; and has broad activity against a variety of tumor cell lines [Gumireddy et al. 2005]. As with other Plk1 inhibitors, it induces mitotic arrest and apoptosis. Two phase I studies with this agent have recently been completed in patients with advanced solid tumors [Advani et al. 2014; Bowles et al. 2014]. A recent paper reported on the use of rigosertib, given as a $72 \mathrm{~h}$ continuous intravenous infusion.in patients with myelodysplastic syndromes (MDS), most with high-risk International Prognostic Scoring System (IPSS) scores, who had previously been treated with hypomethylating agents (HMAs) [Silverman et al. 2014]. Of 40 patients, $12(40 \%)$ achieved a partial or complete marrow blast response, while several others experienced hematologic improvement associated with stable marrow blasts. The drug was well tolerated, with modest myelotoxicity; other toxicities included fatigue, nausea, diarrhea and hematuria (mainly grade 1-2). A phase I study has also been reported using oral rigosertib in high-risk MDS [Komrokji et al. 2013]; notable nonhematologic toxicities included urothelial inflammation, manifest as dysuria and hematuria. As with the previous study, some hematologic responses were observed.

A phase III randomized trial has subsequently been reported with this agent in patients with high-risk MDS who had failed to respond to, relapsed or progressed after, receiving treatment with HMA [Garcia-Manero et al. 2014]. Patients 
Table 1. Summary of Polo-like kinase 1 inhibitors and status of development.

\begin{tabular}{|c|c|c|c|c|c|}
\hline Agent & Company & Regimen & Disease & Phase & Reference \\
\hline GSK4611364A & GlaxoSmithKline & single agent & Solid tumours & I & Olmos \\
\hline TAK-960 & Millennium & single agent & Solid tumours & I & (pending) \\
\hline NMS-P937 & Nerviano & single agent & & & (preclinical) \\
\hline \multirow[t]{4}{*}{ Rigosertib } & Onconova & single agent & Solid tumours & 1 & Bowles, Advani \\
\hline & & single agent & MDS/AML & $1 / I I$ & $\begin{array}{l}\text { Silverman, } \\
\text { Komrokji }\end{array}$ \\
\hline & & single agent & MDS & III & Garcia-Manero \\
\hline & & single agent & AML/ALL/MPD & $1 / I I$ & (pending) \\
\hline $\mathrm{BI} 2536$ & $\begin{array}{l}\text { Boehringer- } \\
\text { Ingelheim }\end{array}$ & single agent & AML & $\mathrm{I} / \mathrm{II}$ & Müller-Tidow \\
\hline \multirow[t]{4}{*}{ Volasertib } & $\begin{array}{l}\text { Boehringer- } \\
\text { Ingelheim }\end{array}$ & single agent & Solid tumours & I & Schöffski \\
\hline & & single agent & AML & $1 / I I$ & Dohner, Bug \\
\hline & & + LDAC & AML & $1 / 11$ & Dohner \\
\hline & & + LDAC & AML & III & (pending) \\
\hline
\end{tabular}

were randomized between rigosertib, given as 72 $\mathrm{h}$ continuous intravenous infusion versus best supportive care. Although this study did not demonstrate a significant difference in OS between the two arms, an improvement in OS in favor of the rigosertib arm was observed within certain subgroups, including those with primary HMA failure (8.6 versus 5.3 months, $p=0.04$ ) and very high-risk revised-IPSS scores.

Several of the early phase studies with rigosertib have included patients with AML, either exclusively or combined with patients with MDS. These have only been reported in abstract form to date, but it has been noted that patients with AML with higher blast percentages were less likely to respond [Navada et al. 2013]. A phase I/ II study in AML, ALL and myeloproliferative disorders has just been completed [ClinicalTrials. gov identifier: NCT01167166], and the results are pending.

\section{Volasertib}

BI6727 (Boehringer-Ingelheim), now called volasertib, is a second in-class dihydropterinone derivative. It is a potent ATP-competitive selective inhibitor of Plk1, and also inhibits Plk2 and Plk3 (IC50 0.87, 5 and $56 \mathrm{nM} / \mathrm{L}$, respectively). This inhibition is highly selective, as it did not show inhibitory activity against over 50 other kinases at clinically relevant concentrations
[Rudolph et al. 2009]. Volasertib has shown activity in vitro against a wide variety of tumor cell lines, including colon, melanoma, lung, lymphoma and AML lines [Rudolph et al. 2009]. Cellular effects are similar to those of other Plk1 inhibitors, with arrest in G2/early $M$ phase and subsequent induction of apoptosis. It also demonstrates broad antitumor activity in vivo in multiple mouse xenograft models.

As with its predecessor compound, the major dose-limiting toxicity in phase I studies in solid tumors has been grade 3-4 reversible myelosuppression [Schöffski et al. 2012]. In addition, transient QTc prolongation is typically seen, but no clinical sequelae of this have been reported to date. Mucositis is infrequent, and no neurotoxicity, cardiotoxicity or nephrotoxicity has been reported.Volasertib is both a substrate and inhibitor of P-glycoprotein (P-gp); therefore, drugdrug interactions with other P-gp substrates are expected but have not yet been described. When administered as a $1 \mathrm{~h}$ intravenous infusion the half life is approximately 110-115 h [Bug et al. 2011; Schöffski et al. 2012].

A phase I study was completed in patients with relapsed/refractory AML [Bug et al. 2011; Döhner et al. 2014a]. When dose escalated as monotherapy in a day 1 and 15 schedule, repeated every 4 weeks, the MTD was determined to be $450 \mathrm{mg}$ [Döhner et al. 2014a]. The dose-limiting toxicity 
at doses of at least $500 \mathrm{mg}$ consisted primarily of mucosal inflammation, with esophagitis and gastrointestinal hemorrhage seen as well. At doses of at least $350 \mathrm{mg}$ antileukemic activity was noted, with 5 of $43(12 \%)$ CRs with incomplete count recovery (CRi) in this heavily pretreated population.

When combined with low-dose cytarabine (LDAC) $20 \mathrm{mg}$ subcutaneously twice daily for 10 days, the MTD of volasertib was $350 \mathrm{mg}$ intravenously given on a day $1+15$ schedule, repeated every 4 weeks [Bug et al. 2011]. The major doselimiting toxicity using this combination was myelosuppression; the CR (CR + CRi) rate in this study was $22 \%$. There was no evidence of drug interactions between volasertib and LDAC.

A subsequent open-label randomized phase II study has been published comparing the combination of volasertib $350 \mathrm{mg}$ intravenously on days 1 and 15 plus LDAC $20 \mathrm{mg}$ subcutaneously twice daily for 10 days on a 28-day cycle, with LDAC alone in the same dosing schedule [Döhner et al. 2014b]. Patients could continue therapy until disease progression. This study enrolled 87 older patients with previously untreated AML who were not candidates for intensive chemotherapy based on physician judgment. The CR (CR + $\mathrm{CRi}$ ) rate for the combination arm was $31 \% \mathrm{com}$ pared with $13 \%$ in the LDAC monotherapy arm. Notably, in patients with adverse risk cytogenetics the $\mathrm{CR}$ rate was $36 \%(5 / 14)$ in the combination arm versus $7 \%(1 / 14)$ in the LDAC monotherapy arm. There was a significantly longer event-free survival $(p=0.021)$ and OS $(p=0.047)$ in the volasertib + LDAC arm. There were more grade 3 adverse events in the combination arm, with respect to febrile neutropenia, documented infections and gastrointestinal events (mainly grade 3 diarrhea), but there was no difference in the 30and 60-day mortality.

Based on these results, a larger ( $>600$ patient) multicenter phase III double-blind randomized placebo-controlled trial was initiated with LDAC \pm volasertib, using the same comparator groups and dosing schedule as in the phase II study [ClinicalTrials.gov identifier: NCT01721876]. Eligible patients included those aged 65 years and over with previously untreated AML who were not considered candidates for intensive chemotherapy, with a $2: 1$ randomization between study drug and. placebo. Accrual to this study has now been completed (as of November 2014), and results are pending.

\section{Summary and future directions}

Plk inhibitors represent a promising new class of anticancer agents. Volasertib is the first agent of its class to be studied clinically, and the first to proceed to phase III testing, in AML. Singleagent activity has been demonstrated in AML, and the combination with low-dose cytarabine is associated with increased antileukemic activity compared with cytarabine alone, with an acceptable toxicity profile. The current phase III study will determine whether OS is improved using this combination.

Given its limited nonhematologic toxicity, volasertib is also an attractive agent to consider in other combination regimens. A phase I study is planned combining this agent with induction chemotherapy in AML. Further preclinical studies are also warranted, evaluating potential synergy with other agents. Synergy with vincristine has been seen in leukemia cell lines [Ikezoe $e t$ al. 2009]. Exploratory studies combining these drugs with hypomethylating agents would also be worth considering in view of the activity and tolerability of both agents and their nonoverlapping mechanisms of action. Plk1 inhibitors may also have a potential role as maintenance therapy, particularly in older patients with AML who are not transplant candidates.

Other Plk1 inhibitors also warrant testing in AML. As mentioned, rigosertib has demonstrated activity in high-risk MDS. It may offer an advantage of limited myelotoxicity at the doses used, but its use may be limited by nonhematologic bladder toxicity. The continuous infusion schedule may offer additional theoretical advantages, by hitting a higher proportion of leukemic cells as they enter mitosis; its dual mechanism of action may produce additional antileukemic activity. Although it appears to have limited activity in AML as a single agent, it may have greater activity in combination. Some of the newer agents such as NMS-P937 have higher selectivity for Plk1 and a lower IC50, which may result in different efficacy and toxicity profiles. The oral Plk1 inhibitors, including TAK960 and NMS-P937, would offer a potential advantage of convenience, particularly in the maintenance setting, in older patients. 


\section{Funding}

This research received no specific grant from any funding agency in the public, commercial or notfor-profit sectors.

\section{Conflict of interest statement}

The author has received research funding from Boehringer-Ingelheim, Merck and Novartis for clinical trials, and has participated in advisory boards for Celgene, Lundbeck and Boehringer-Ingelheim.

\section{References}

Advani, S., Achrekar, S., Doval, D., Raghunadharao, D., Wilhelm, F. and Acharya, M. (2014) Phase 1 dose escalation study of rigosertib by 2-, 4-, or 8-hour infusion twice-weekly in patients with advanced cancer. Indian $\mathcal{F}$ Cancer 51: 40-44.

Bowles, D., Diamond, J., Lam, E., Weekes, C., Astling, D., Anderson, R. et al. (2014) Phase I study of oral rigosertib (ON 01910.Na), a dual inhibitor of the PI3K and PLK1 pathways, in adult patients with advanced solid malignancies. Clin Cancer Res 20: 1656-1665.

Bug, G., Müller-Tidow, C., Schlenk, R., Krämer, A., Lübbert, M., Krug, U. et al. (2011) Phase I/II study of volasertib (BI 6727), an intravenous pololike kinase (PLK) inhibitor, in patients with acute myeloid leukemia (AML): updated results of the dose finding phase I part for volasertib in combination with lowdose cytarabine (LD-Ara-C) and as monotherapy in relapsed/refractory AML. Blood 118(21): abstract 1549.

Burnett, A., Milligan, D., Prentice, A., Goldstone, A., McMullin, M., Hills, R. and Wheatley, K. (2007) A comparison of low-dose cytarabine and hydroxyurea with or without all-trans retinoic acid for acute myeloid leukemia and high-risk myelodysplastic syndrome in patients not considered fit for intensive treatment. Cancer 109: 1114-1124.

Casolaro, A., Golay, J., Albanese, C., Ceruti, R., Patton, V., Cribioli, S. et al. (2013) The polo-like kinase 1 (PLK1) inhibitor NMS-P937 Is effective in a new model of disseminated primary CD56+ acute monoblastic leukaemia. PLoS One 8: e58424.

Chopra, P., Sethi, G., Dastidar, A. and Ray, A. (2010) Polo-like kinase inhibitors: an emerging opportunity for cancer therapeutics. Expert Opin Investig Drugs 19: 27-43.

Craig, S., Wyatt, M. and McInnes, C. (2014) Current assessment of pol-like kinases as anti-tumor drug targets. Expert Opin Drug Discov 9: 773-789.

Dai, W., Wang, Q. and Traganos, F. (2002) Pololike kinases and centrosome regulation. Oncogene 21: 6195-6200.

Döhner, H., Bug, G., Müller-Tidow, C., Krämer, A., Lübbert, M, Krug, U. et al. (2014a) Phase I/II study of volasertib, an intravenous polo-like kinase inhibitor (PLK), in patients with relapsed/refractory acute myeloid leukemia (AML): updated phase I results for volasertib monotherapy. Haematologica 99(suppl. 1): abstract S649.

Döhner, H., Lübbert, M., Fiedler, W., Fouillard, L., Haaland, A., Brandwein, J. et al. (2014b) Lowdose cytarabine with or without volasertib (BI 6727), a polo-like kinase inhibitor, in patients with newly diagnosed acute myeloid leukemia not considered suitable for intensive induction therapy. A randomized phase II trial. Blood 124: 1426-1433.

Dombret, H., Seymour, J., Butrym, A., Wierzbowska, A., Selleslag, D., Jang, J. et al. (2014) Results of a phase 3, multicentre, randomized, open-label study of azacitidine (Aza) vs conventional care regimens (CCR) in older patients with newly diagnosed acute myeloid leukemia (AML). Haematologica 99(suppl. 1): abstract LB-6212.

Farag, S., Archer, K., Mrozek, K., Ruppert, A., Carroll, A., Vardiman, J. et al. (2006) Pretreatment cytogenetics add to other prognostic factors predicting complete remission and long-term outcome in patients age 60 years or older with acute myeloid leukemia: results from the Cancer and Leukemia Group B 8461. Blood 108: 63-73.

Fenaux, P., Mufti, G., Hellstrom-Lindberg, E., Santini, V., Gattermann, N., Germing, U. et al. (2010) Azacitidine prolongs overall survival compared with conventional care regimens in elderly patients with low bone marrow blast count acute myeloid leukemia. $\mathcal{F}$ Clin Oncol 28: 562-569.

Garcia-Manero, G., Fenaux, P., Al-Kali, A., Baer, M., Sekeres, M., Roboz, G. et al. (2014) Overall survival and subgroup analysis from a randomized phase III study of intravenous rigosertib versus best supportive care (BSC) in patients with higher-risk myelodysplastic syndrome (HR-MDS) after failure of hypomethylating agents (HMAs). Blood 124(21): abstract 163.

Grimwade, D., Hills, R., Moorman, A., Walker, H., Chatters, S., Goldstone, A. et al. (2010) Refinement of cytogenetic classification in acute myeloid leukemia: determination of prognostic significance of rare recurring chromosome abnormalities among 5876 younger adult patients treated in the United Kingdom Medical Research Council trials. Blood 116: 354-365.

Grimwade, D., Walker, H., Harrison, G., Oliver, F., Chatters, S., Harrison, C. et al. (2001) The predictive value of hierarchical cytogenetic classification in older adults with acute myeloid leukemia: analysis of 1065 patients entered into the United Kingdom Medical research Council AML11 trial. Blood 98: 1312-1320.

Gumireddy, K., Ramana Reddy, M., Cosenza, S., Boominathan, R., Baker, S., Papathi, N. et al. (2005) ON01910, a non-ATP-competitive small molecule inhibitor of PLK1, is a potent anticancer agent. Cancer Cell 7: 275-286. 
Gupta, V., Chun, K., Yi, Q.-L., Minden, M., Schuh, A., Wells, R. et al. (2005) Disease biology rather than age is the most important determinant of survival of patients $>$ or $=60$ years with acute myeloid leukemia treated with uniform intensive therapy. Cancer 103: 2082-2090.

Hansen, D., Loktev, A., Ban, K. and Jackson, P. (2004) PLK1 regulates activation of the anaphase promoting complex by phosphorylating and trigerring SCFbeta-TrCP dependant destruction of the APC inhibitor Dmi1. Mol Biol Cell 15: 5623-5634.

Harousseau, J., Martinelli, G., Jedrzejczak, W., Brandwein, J., Bourdessoule, D., Masszi, T. et al. (2009) A randomized phase 3 study of tipifarnib compared to best supportive care, including hydroxyurea, in the treatment of newly diagnosed acute myeloid leukemia (AML) in patients 70 years or older. Blood 114: 1166-1173.

Hikichi, Y., Honda, K., Hikami, K., Miyashita, H., Kaieda, I., Mural, S. et al. (2012) TAK-960, a novel, orally available, selective inhibitor of polo-like kinase 1, shows broad-spectrum preclinical antitumor activity in multiple dosing regimens. Mol Cancer Ther 11: 700-709.

Hofheinz, R., Al-Batran, S., Hochhaus, A., Jäger, E., Reichardt, V., Fritsch, H. et al. (2010) An open-label, phase I study of the polo-like kinase-1 inhibitor, BI 2536, in patients with advanced solid tumors. Clin Cancer Res 16: 4666-4674.

Ikezoe, T., Yang, J., Nishioka, C., Takezaki, Y., Tasaka, T., Togitani, K. et al. (2009) A novel treatment strategy targeting polo-like kinase 1 in hematological malignancies. Leukemia 23: 1564-1576.

Kantarjian, H., O’Brien, S., Cortes, J., Giles, F., Faderl, S., Jabbour, E. et al. (2006) Results of intensive chemotherapy in 998 patients age 65 years or older with acute myeloid leukemia or high-risk myelodysplastic syndrome: predictive prognostic models for outcome. Cancer 106: 1090-1098.

Kantarjian, H., Thomas, X., Dmoszynska, A., Wierzbowska, A., Mazur, G., Mayer, J. et al. (2012) Multicenter, randomized, open-label, phase III trial of decitabine versus patient choice, with physician advice, of either supportive care or low-dose cytarabine for the treatment of older patients with newly diagnosed acute myeloid leukemia. $7 \mathrm{Clin}$ Oncol 30: 2670-2677.

Knecht, R., Oberhauser, C. and Strebhardt, K. (2000) PLK (polo-like kinase), a new prognostic marker for oropharyngeal carcinomas. Int $\mathcal{F}$ Cancer 89: 535-536.

Komrokji, R., Raza, A., Lancet, J., Ren, C., Taft, D., Maniar, M. et al. (2013) Phase I clinical trial of oral rigosertib in patients with myelodysplastic syndromes. Br f Haematol 162: 417-524.
Lenart, P., Petronczki, M., Steegmaier, M., Di Fiore, B., Lipp, J., Hoffmann, M. et al. (2007) The small molecule inhibitor BI 2536 reveals novel insights into mitotic roles polo-like kinase 1. Curr Biol 17: 304-315.

Linch, D., Hills, R., Burnett, A., Khwaja, A. and Gale, R. (2014) Impact of FLT3(ITD) mutant allele level on relapse risk in intermediate-risk acute myeloid leukemia. Blood 124: 273-276.

Liu, L., Zhang, M. and Zou, P. (2007) Expression of PLK1 and survivin in diffuse large B-cell lymphoma. Leuk Lymphoma 48: 2179-2183.

Liu, X. and Erikson, R.L. (2003) Polo-like kinase (PLK) 1 depletion induces apoptosis in cancer cells. Proc Natl Acad Sci U S A 100: 5789-5794.

Mrozek, K., Marcucci, G., Nicolet, D., Maharry, K., Becker, H., Whitman, S. et al. (2012) Prognostic significance of the European LeukemiaNet standardized system for reporting cytogenetic and molecular alterations in adults with acute myeloid leukemia. f Clin Oncol 30: 4515-4523.

Müller-Tidow, C., Bug, G., Lübbert, M., Krämer, A., Krauter, J., Valent, P. et al. (2013) A randomized, open-label, phase I/II trial to investigate the maximum tolerated dose of the Polo-like kinase inhibitor BI 2536 in elderly patients with refractory/relapsed acute myeloid leukaemia. Br F Haematol 163: 214-222.

Navada, S., Odchimar-Reissig, R., Reddy, E., Demakos, E., Holland, J., Wilhelm, F. et al. (2013) Predictors of response to rigosertib in patients with a myelodysplastic syndrome (MDS) or acute myeloid leukemia (AML) relapsing after or refractory to hypomethylating agents. Blood 122(21): abstract 633.

Olmos, D., Barker, D., Sharma, R., Brunetto, A., Yap, T., Taegtmeyer, A. et al. (2011) Phase I study of GSK461364, a specific and competitive Polo-like kinase 1 inhibitor, in patients with advanced solid malignancies. Clin Cancer Res 17: 3420-3430.

Pemmaraju, N., Kantarjian, H., Garcia-Manero, G., Pierce, S., Cardenas-Turanzas, M., Cortes, J. et al. (2015) Improving outcomes for patients with acute myeloid leukemia in first relapse: a single center experience Am F Hematol 90: 27-30.

Renner, A., Dos Santos, C., Recher, C., Bailly, C., Créancier, L., Kruczynski, A. et al. (2009) Pololike kinase 1 is overexpressed in acute myeloid leukemia and its inhibition preferentially targets the proliferation of leukemic cells. Blood 114: 659-662.

Rudolph, D., Steegmaier, M., Hoffmann, M., Grauert, M., Baum, A., Quant, J. et al. (2009) BI 6727, a polo-like kinase inhibitor with improved pharmacokinetic profile and broad antitumor activity. Clin Cancer Res 15: 3094-3102. 
Schnittger, S., Schoch, C., Kern, W., Mecucci, C., Tschulik, C., Martelli, M. et al. (2005) Nucleophosmin gene mutations are predictors of favorable prognosis in acute myelogenous leukemia with a normal karyotype. Blood 106: 3733-3739.

Schöffski, P., Awada, A., Dumez, H., Gil, T., Bartholomeus, S., Wolter, P. et al. (2012) A phase I, dose-escalation study of the novel Polo-like kinase inhibitor volasertib (BI 6727) in patients with advanced solid tumours. Eur F Cancer 48: 179-186.

SEER (2014) Cancer Statistics Review 1975-2004. Available at: http://seer.cancer.gov (accessed 22 January 2015).

Silverman, L., Greenberg, P., Raza, A., Olnes, M., Holland, J., Reddy, P. et al. (2014) Clinical activity and safety of the dual pathway inhibitor rigosertib for higher risk myelodysplastic syndromes following DNA methyltransferase inhibitor therapy. Hematol Oncol doi:10.1002/hon.2137.

Steegmaier, M., Hoffmann, M., Baum, A., Lénárt, P., Petronczki, M., Krššák, M. et al. (2007) BI 2536, a potent and selective inhibitor of polo-like kinase 1, inhibits tumor growth in vivo. Curr Biol 17: 316-322.

Strebhardt, K. (2010) Multifaceted polo-like kinases: drug targets and antitargets for cancer therapy. Nat Rev Drug Discov 9: 643-660.

Strebhardt, K., Kneisel, L., Linhart, C., Bernd, A. and Kaufmann, R. (2000) Prognostic value of pololike kinase expression in melanomas. $\mathcal{F A M A} 283$ : 479-480.

Tallman, M., Gilliland, D. and Rowe, J. (2005)

Drug therapy for acute myeloid leukemia. Blood 106: 1154-1163.

Tamura, Y., Simizu, S., Muroi, M., Takagi, S., Kawatani, M., Watanabe, N. et al. (2009) Polo-like kinase 1 phosphorylates and regulates $\mathrm{Bcl}-\mathrm{x}(\mathrm{L})$ during pironetin-induced apoptosis. Oncogene 28: 107-116.

Valsasina, B., Beria, I., Alli, C., Alzani, R., Avanzi, N., Ballinari, D. et al. (2012) NMS-P937, an orally available, specific small-molecule polo-like kinase 1 inhibitor with antitumor activity in solid and hematologic malignancies. Mol Cancer Ther 11: 1006-1016.

van Gelder, M., de Wreede, L., Schetelig, J., van Biezen, A., Volin, L., Maeterns, J. et al. (2013) Monosomal karyotype predicts poor survival after allogeneic stem cell transplantation in chromosome 7 abnormal myelodysplastic syndrome and acute myeloid leukemia. Leukemia 27: 879-888.

van Vugt, M., van de Weerdt, B., Vader, G., Janssen, H., Calafat, J., Klompmaker, R. et al. (2004) Polo-like kinase- 1 is required for bipolar spindle formation but is dispensible for anaphase promoting complex Cdc20 activation and initiation of cytokinesis. F Biol Chem 279: 36841-36854.

Weichert, W., Denkert, C., Schmidt, M., Gekeler, V., Wolf, G., Köbel, M. et al. (2004) Polo-like kinase isoform expression is a prognostic factor in ovarian carcinoma. Br f Cancer 90: 815-821.

Winkles, J. and Alberta, G. (2005) Differential regulation of polo-like kinase 1, 2, 3, and 4 gene expression in mammalian cells and tissues. Oncogene 24: 260-266.

Wolf, G., Elez, R., Doermer, A., Holtrich, U., Ackermann, H., Stutte, H. et al. (1997) Prognostic significance of polo-like kinase (PLK) expression in non-small cell lung cancer. Oncogene 14: 543-549.

Wolf, G., Hildenbrand, R., Schwar, C., Grobholz, R., Kaufmann, M., Stutte, H. et al. (2000) Polo-like kinase: a novel marker of proliferation: correlation with estrogen-receptor expression in human breast cancer. Pathol Res Pract 196: 753-759.
Visit SAGE journals online http://tah.sagepub.com

@SAGE journals 